\title{
Abemaciclib, a CDK4 and CDK6 inhibitor for the treatment of metastatic breast cancer
}

\author{
Miguel Martin*,1(D), Jose A Garcia-Saenz ${ }^{2}$, Luis Manso ${ }^{3}$ (D) Antonio Llombart ${ }^{4}$, Alejo \\ Cassinello ${ }^{5}$, Manuel Atienza ${ }^{5}$, Francois Ringeisen ${ }^{6}$ \& Eva Ciruelos ${ }^{7}$ (D) \\ ${ }^{1}$ Gregorio Marañon Health Research Institute, Universidad Complutense, CIBERONC, Dr. Esquerdo 46, 28006, Madrid, Spain \\ ${ }^{2}$ Medical Oncology Department, Hospital Clínico San Carlos Health Research Institute (IdISSC), 28040, Madrid, Spain \\ ${ }^{3}$ Medical Oncology Department, 12 de Octubre University Hospital, Avda Andalucia s/n., 28041, Madrid, Spain \\ ${ }^{4}$ Medical Oncology Department, Arnau de Vilanova Hospital, FISABIO, Universidad Católica Valencia, San Clemente 12, \\ 46015, Valencia, Spain \\ ${ }^{5}$ Eli Lilly \& Company, Av. de la Industria 30, 28108, Alcobendas, Spain \\ ${ }^{6}$ Eli Lilly Export SA, 16, Ch. Des Coquelicots, CH-1214, Vernier, Switzerland \\ ${ }^{7}$ Breast Cancer Unit, Medical Oncology Department, 12 de Octubre University Hospital, HM CIOCC, 28041, Madrid, Spain \\ *Author for correspondence: mmartin@geicam.org
}

The addition of CDK4 and 6 inhibitors (abemaciclib, palbociclib or ribociclib) to endocrine therapy, as firstline treatment or following progression after initial endocrine therapy, significantly increased progressionfree survival, objective response rates and in some trials overall survival, compared with endocrine therapy alone in HR+ and HER2- breast metastatic breast cancer. These CDK4 and 6 inhibitors are now approved in this context and have become a new standard of care. A hypothesis-generating exploratory analysis suggested that the addition of abemaciclib to endocrine therapy showed the largest effects in subgroups of women with indicators of poor prognosis, although these data require confirmation. This review provides updated clinical trial data for all three drugs in metastatic breast cancer, focusing on abemaciclib, the most recently approved agent.

First draft submitted: 11 June 2020; Accepted for publication: 23 July 2020; Published online: 12 August 2020

Keywords: abemaciclib $\bullet$ breast cancer $\bullet$ CDK4/6 inhibitors $\bullet$ hormone receptor-positive $\bullet$ human epidermal growth factor receptor 2-negative $\bullet$ metastatic

HR+ and HER2- breast cancer represents approximately two-thirds of all breast cancers $[1,2]$ and hormonal therapy is key in its management [3]. However, primary or acquired hormonal resistance limits strategies targeting the estrogen receptor axis. Most $\mathrm{HR}+$ metastatic breast cancers initially sensitive to hormonal therapy ultimately display resistance [3]. Adaptive mechanisms related to endocrine resistance include activation of signaling pathways and alteration of survival and cell cycle pathways. Combinations of endocrine therapy with targeted agents thus aim to reverse or delay endocrine resistance [3].

CDK4 and 6 have gained increasing attention in HR+ breast cancer [4-8]. CDK4 and 6 promote cell cycle transition through the G1 restriction point to the $S$ phase where DNA replication occurs. CDK 4 and 6 operate with their respective $\mathrm{D}$-type cyclin partners and these complexes are regulated by small inhibitory proteins [9]. The CDK4-cyclin D and CDK6-cyclin D pathways are frequently altered in human cancers [4-6].

Three orally administered CDK4 and 6 inhibitors are available for the treatment of advanced or metastatic breast cancer: abemaciclib (verzenio ${ }^{\circledR}$; Eli Lilly and Company, Ltd., IN, USA), palbociclib (ibrance ${ }^{\circledR}$; Pfizer, Inc., NY, USA) and ribociclib (kisqali ${ }^{\circledR}$; Novartis, Basel, Switzerland). This article provides an overview of clinical trial data for CDK4 and 6 inhibitors in metastatic breast cancer, focusing on abemaciclib, the most recently approved agent. It describes the molecules' respective pharmacologic characteristics, administration schedules, efficacy in combination with endocrine agents, single-agent activity, tolerability profiles and other characteristics based on available data.

Future Medicine 


\section{Clinical trials with palbociclib, ribociclib \& abemaciclib in HR+/HER2- advanced breast cancer Palbociclib}

Three randomized trials have evaluated palbociclib in patients with advanced breast cancer [10-12]. PALOMA-1, an open-label Phase II study, showed significantly longer median progression-free survival (PFS) with palbociclib plus letrozole than with letrozole alone (20.2 vs 10.2 months; hazard ratio [HR]: 0.488 ; 95\% CI: 0.319-0.748; $\mathrm{p}=0.0004)$ as initial treatment in postmenopausal women with HR+/HER2- advanced breast cancer [10]. Two randomized, double-blind Phase III trials (PALOMA-2 and PALOMA-3) then investigated the efficacy and tolerability of combination palbociclib therapy $[11,12]$.

PALOMA-2 [11] evaluated palbociclib plus letrozole versus placebo plus letrozole in 666 postmenopausal women with a similar general clinical profile as the 165 study participants in PALOMA-1 [10]. In PALOMA-2, median PFS was 24.8 months with palbociclib plus letrozole compared with 14.5 months with placebo plus letrozole; results significantly favored the palbociclib-containing treatment arm (HR: 0.58; 95\% CI: 0.46-0.72; p < 0.001) [11].

After a median follow-up of 38 months, median PFS was 27.6 months with palbociclib plus letrozole and 14.5 months with placebo plus letrozole in the overall population (HR: 0.56; $<<0.0001$ ) [13]. With approximately an additional 15 months of follow-up, palbociclib plus letrozole continued to demonstrate improved PFS in the overall population and across subgroups evaluated and had a favorable safety profile. Overall survival (OS) data for PALOMA-2 had not been reported at the time of writing. Regarding health-related quality of life (HRQoL) patient-reported outcomes, no statistically significant differences were observed between the treatment arms in change from baseline scores for any of the subscales assessed [13].

PALOMA-3 was conducted in 521 pre- or postmenopausal women with HR+/HER2- metastatic breast cancer who had progressed on previous endocrine therapy [12]. Approximately one-third of patients received one line of chemotherapy for advanced breast cancer. Median PFS was significantly improved with palbociclib plus fulvestrant versus placebo plus fulvestrant, with a median PFS of 9.5 months (95\% CI: 9.2-11.0) versus 4.6 months (95\% CI: 3.5-5.6), respectively (HR: 0.46; 95\% CI: 0.36-0.59; $<<0.0001$ ). Numerical increases in median OS, although not statistically significant at the prespecified level, were shown (34.9 vs 28.0 months, respectively; HR: 0.81; 95\% CI: 0.64-1.03; p = 0.09) [14,15]. Estimated overall global HRQoL scores significantly favored palbociclib plus fulvestrant, as did improvement from baseline in pain [16].

In PALOMA-1, -2 and -3, the proportion of patients with objective response rate (ORR) with measurable disease (a secondary end point) was statistically greater with palbociclib plus endocrine therapy than with placebo plus endocrine therapy [10-12]. In PALOMA-1, objective response was achieved in a significantly greater proportion of patients receiving palbociclib plus letrozole than placebo plus letrozole in the population with measurable disease (55\% [95\% CI: 43-68] vs 39\% [95\% CI: 28-52]; p = 0.047) [10]. Patients in PALOMA-2 and -3 with measurable disease also had significantly higher ORRs with palbociclib plus endocrine therapy versus endocrine therapy alone. In PALOMA-2, an ORR was reported in 55.3\% (95\% CI: 49.9-60.7) of patients versus 44.4\% (95\% CI: 36.952.2) (OR: 1.55; 95\% CI: 1.05-2.28; $\mathrm{p}=0.03$ ) [11]. Corresponding ORRs in PALOMA-3 were 25\% (95\% CI: 19.6-30.2) versus $11 \%$ (95\% CI: 6.2-17.3) (OR 2.69; 95\% CI: 1.43-5.26; $\mathrm{p}=0.0012)$ [12]. Only PALOMA-3 also showed a statistically significant advantage with palbociclib for ORR in the intent-to-treat (ITT) population. In all three PALOMA trials, the primary end point was investigator-assessed PFS and analysis was by ITT [10-12].

In recent exploratory analyses of treatment effect by intrinsic and prognostic subtypes in PALOMA-2 and -3 , the addition of palbociclib to endocrine therapy significantly increased PFS regardless of luminal subtype (A or B) and regardless of length of initial treatment-free interval (i.e., time between end of any neoadjuvant or adjuvant therapy and relapse) in PALOMA-2 or disease-free interval (i.e., time between first diagnosis of breast cancer and disease recurrence) in PALOMA-3 [17]. In the PALOMA-3 trial, palbociclib was associated with an increase in median OS, however, non-significant [14]. A planned subgroup analysis of OS regarding the three prespecified stratification factors showed that palbociclib seemed to improve OS in patients with endocrine-sensitive cancers, whereas those with intrinsic endocrine resistance apparently had no OS benefit [14].

The most frequently reported adverse events (AEs) with palbociclib (all-grade incidence $\geq 10 \%$ ) are neutropenia, infections, leukopenia, fatigue, nausea, stomatitis and anemia [18]. Approximately two-thirds of palbociclib recipients in PALOMA-2 and -3 experienced grade $\geq 3$ neutropenia $[11,14,18]$. 


\section{Ribociclib}

Ribociclib has been evaluated in three Phase III randomized trials (MONALEESA-2, -3 and -7) in women with HR+/HER2- advanced breast cancer, with the primary end point being investigator-assessed PFS in the ITT population. As with palbociclib, ribociclib was intermittently dosed: 3 weeks on then 1 week off.

MONALEESA-2 was conducted in 668 postmenopausal women with HR+/HER2- recurrent or metastatic breast cancer who had not previously received systemic therapy for advanced disease [19]. After a first interim analysis showed longer PFS in the ribociclib arm [19], a second interim analysis demonstrated a median PFS of 25.3 months (95\% CI: 23.0-30.3) for ribociclib plus letrozole compared with 16.0 months (95\% CI: 13.4-18.2) for placebo plus letrozole (HR: 0.568; 95\% CI: 0.457-0.704; $\mathrm{p}=9.63 \times 10^{-8}$ ) [20]. OS data for MONALEESA-2 have not yet been reported at the time of writing. Evaluation of HRQoL showed a clinically meaningful improvement in European Organisation for Research and Treatment of Cancer (EORTC) Quality of Life Questionnaire (QLQ)-C30 global health status/QoL scores in both treatment groups at some timepoints and differences between treatment arms in overall HRQoL were less than predefined minimally important differences [21]. Improvements in EORTC QLQC30 pain scores were observed as early as week 8 , with clinically meaningful reductions from day 1 of cycle 3-15 in the ribociclib arm and at cycles 7 and 15 only in the placebo plus letrozole arm [21].

In MONALEESA-7, 672 premenopausal women with HR+/HER2- advanced breast cancer were randomized to receive ribociclib or placebo, both with goserelin and either tamoxifen or a nonsteroidal aromatase inhibitor (NSAI) [22]. Previous endocrine therapy was permitted in the neoadjuvant and adjuvant setting, as was chemotherapy in the neo/adjuvant setting or for advanced disease. Median PFS was significantly longer with ribociclib (23.8 months; 95\% CI: 19.2-not reached) than with placebo (13.0 months; 95\% CI: 11.0-16.4) (HR: 0.55; 95\% CI: $0.44-0.69 ; \mathrm{p}<0.0001$ ) [22]. An interim analysis for OS, a secondary end point of the study, showed that adding ribociclib to endocrine therapy resulted in significantly longer OS than endocrine therapy alone [23]. The estimated OS at 42 months was 70.2\% (95\% CI: 63.5-76.0) with ribociclib and 46\% (95\% CI: 32.0-58.9) with placebo (HR for death: $0.71 ; 95 \%$ CI: $0.54-0.95 ; \mathrm{p}=0.00973$ by log-rank test).

MONALEESA-3 compared ribociclib plus fulvestrant versus placebo plus fulvestrant in 726 postmenopausal women with HR+/HER2- advanced breast cancer who were treatment naive or had received up to one line of prior endocrine therapy in the advanced setting [24]. Median PFS was 20.5 months (95\% CI: 18.5-23.5) versus 12.8 months (95\% CI: 10.9-16.3), respectively, (HR: 0.593; 95\% CI: 0.480-0.732; p < 0.001) [24]. Treatment effects were consistent for treatment-naive patients (HR: 0.577 ; 95\% CI: 0.415-0.802) and those who had received up to one line of prior endocrine therapy (HR: 0.565; 95\% CI: 0.428-0.744) in the advanced setting [24]. There were no statistically significant differences between groups for changes in HRQoL, evaluated using the EORTC QLQ-C30 (assessing global health status and HRQoL) and the Brief Pain Inventory-short form questionnaire (assessing pain) and mean global health scores improved or did not deteriorate from baseline during every cycle of treatment in both arms [25]. In the second OS analysis, the study also met its key secondary end point, demonstrating a statistically significant improvement in OS that was consistent across all subgroups. ribociclib in combination with fulvestrant showed a $28 \%$ reduction in risk of death in the ITT population (median OS not reached vs 40.0 months; HR: 0.724; 95\% CI: 0.568-0.924; $\mathrm{p}=0.00455$ ) [26].

The MONALEESA trials also showed that ORR, a secondary end point of all three studies, was significantly greater with ribociclib plus endocrine therapy [19,22,24].

Subgroup analyses of three pivotal Phase III MONALEESA trials showed ribociclib plus endocrine therapy extended PFS in all patients with and without visceral involvement compared with endocrine therapy alone; this was consistent with the overall study populations [27].

Across studies, the most frequently reported all-grade AEs with ribociclib $(\geq 20 \%)$ were neutropenia, nausea, fatigue, diarrhea and leukopenia [28]. The frequency of these all-grade AEs was $\geq 2 \%$ higher with ribociclib than with placebo in at least one of the MONALEESA trials $[19,22,24,28]$. Grade 3 or 4 AEs reported at a frequency $\geq 5 \%$ with ribociclib in all three trials included neutropenia, leukopenia and increased ALT and/or AST [28]. In MONALEESA-2, grade 3 or 4 AEs reported in $\geq 10 \%$ of patients included neutropenia ( 59.3 vs $0.9 \%$ with placebo) and leukopenia (21.0 vs 0.6\%) [19]. In MONALEESA-2 and -3, concurrent elevations in alanine aminotransferase or aspartate aminotransferase $>3$ times the upper limit of normal and total bilirubin $>2$ times the upper limit of normal, with normal alkaline phosphatase, in the absence of cholestasis occurred in six patients $(\approx 1 \%)$ [28]. Across MONALEESA-2, -7 and -3 , approximately $6 \%$ of ribociclib recipients had a $>60 \mathrm{~ms}$ increase from baseline in QT interval corrected by fridericia's formula (QTcF) [28]. 


\section{Abemaciclib}

Abemaciclib has been evaluated in two Phase III trials (MONARCH 3 and 2) [29,30] and an earlier Phase II trial (MONARCH 1) [31] in women with advanced or metastatic HR+/HER2- breast cancer (Table 1). Abemaciclib was administered twice daily on a continuous dosing schedule. Table 2 shows ongoing trials with abemaciclib in breast cancer (clinicaltrials.gov).

MONARCH 3 was a randomized, double-blind, placebo-controlled, multinational study evaluating the efficacy of adding abemaciclib to NSAI therapy in postmenopausal women with HR+/HER2- locoregionally recurrent or metastatic breast cancer who had not previously received systemic treatment in this setting [29]. Among other inclusion criteria were an Eastern Cooperative Oncology Group (ECOG) performance status of zero or one and either measurable disease or nonmeasurable bone-only disease as defined by Response Evaluation Criteria in Solid Tumors (RECIST) v1.1. Patients with a history of brain metastases and previous treatment with any CDK4 and 6 inhibitor were excluded.

Patients in MONARCH 3 were randomized 2:1 to receive either oral abemaciclib $150 \mathrm{mg}$ every $12 \mathrm{~h}$ or placebo, in addition to oral NSAI treatment with either anastrozole $1 \mathrm{mg}$ once daily or letrozole $2.5 \mathrm{mg}$ once daily. The primary end point of the trial was investigator-assessed PFS in the ITT population.

A planned interim analysis was conducted after 189 PFS events in 493 women, which included 328 and 165 in the respective treatment groups [29]. Abemaciclib plus NSAI was effective as initial therapy, improving PFS and ORR, with a generally tolerable safety profile [29]. Median PFS was significantly prolonged with abemaciclib plus NSAI versus placebo plus NSAI (HR: 0.54; 95\% CI: 0.41-0.72; $\mathrm{p}=0.000021$ ), thereby meeting the primary end point of the study and was consistent across subgroups. Abemaciclib plus NSAI was also associated with a significantly better ORR than placebo plus NSAI in women with measurable disease $(59.2$ vs $43.8 \%$; $\mathrm{p}=0.004)$.

The updated preplanned final results from MONARCH 3 were recently reported after a median follow-up period of 26.7 months [32,35]. Median PFS was 28.2 months with abemaciclib plus NSAI versus 14.8 months with placebo plus NSAI (HR: 0.540; 95\% CI: 0.418-0.698; $\mathrm{p}=0.000002$ ) (Figure 1).

A recent analysis of MONARCH 3 data evaluated the intermediate end points time to subsequent chemotherapy (TTC) and time to second disease progression or death (PFS2) as a bridge between PFS and OS [36. In the ITT population, $21.3 \%$ of abemaciclib recipients and $36.4 \%$ of placebo recipients received chemotherapy after discontinuing study treatment. Abemaciclib-treated women had statistically significant improvements in TTC (HR: 0.539; 95\% CI: 0.381-0.763; p < 0.001) and PFS2 (HR: 0.735; 95\% CI: 0.545-0.991; p = 0.0426) [36]. OS data for MONARCH 3 had not been reported at the time of writing.

MONARCH 2 was a randomized, double-blind, placebo-controlled, multinational, Phase III study comparing the efficacy and safety of abemaciclib plus fulvestrant versus fulvestrant alone in a 2:1 ratio in pre- or postmenopausal women with HR+/HER2- locally advanced or metastatic breast cancer who had progressed while receiving endocrine therapy [30]. Disease progression could have occurred while receiving neoadjuvant or adjuvant endocrine therapy, up to 12 months from the end of adjuvant endocrine therapy or while receiving first-line endocrine therapy for metastatic disease. Among other inclusion criteria were an ECOG performance status of zero or one, either measurable disease (RECIST v.1.1) or nonmeasurable bone-only disease and postmenopausal status due to either surgical/natural menopause or ovarian suppression. Exclusion criteria included a history of brain metastases and previous chemotherapy (other than adjuvant/neoadjuvant chemotherapy), fulvestrant, everolimus, or any CDK4 and 6 inhibitor.

Initially, abemaciclib was administered at a continuous dosage regimen of $200 \mathrm{mg}$ orally every $12 \mathrm{~h}$, then reduced to $150 \mathrm{mg}$ every $12 \mathrm{~h}$ after a review of safety data and dose reduction rates. Fulvestrant $500 \mathrm{mg}$ was administered intramuscularly on days 1 and 15 of the first cycle, then on day 1 of cycle 2 and beyond. The primary end point was investigator-assessed PFS in the ITT population.

Patients $(n=669)$ were randomized to receive abemaciclib plus fulvestrant $(n=446)$ or placebo plus fulvestrant $(\mathrm{n}=223)$. The median length of follow-up was 19.5 months [30]. Median PFS was significantly longer with abemaciclib plus fulvestrant than with placebo plus fulvestrant (16.4 vs 9.3 months; HR: 0.553 ; 95\% CI: 0.449$0.681 ; \mathrm{p}<0.001$ ) (Figure 1), thereby meeting the primary end point. PFS was extended across patient subgroups [30]. Consistent with the primary analysis, updated PFS data from a follow-up analysis showed significantly longer median PFS with the addition of abemaciclib to fulvestrant (16.9 vs 9.3 months; HR: 0.536; 95\% CI: 0.445-0.645). This analysis also showed significantly longer OS (a key secondary end point) with abemaciclib plus fulvestrant than with placebo plus fulvestrant [33]. After a median follow-up time of 47.7 months, the estimated OS was 46.7 months 


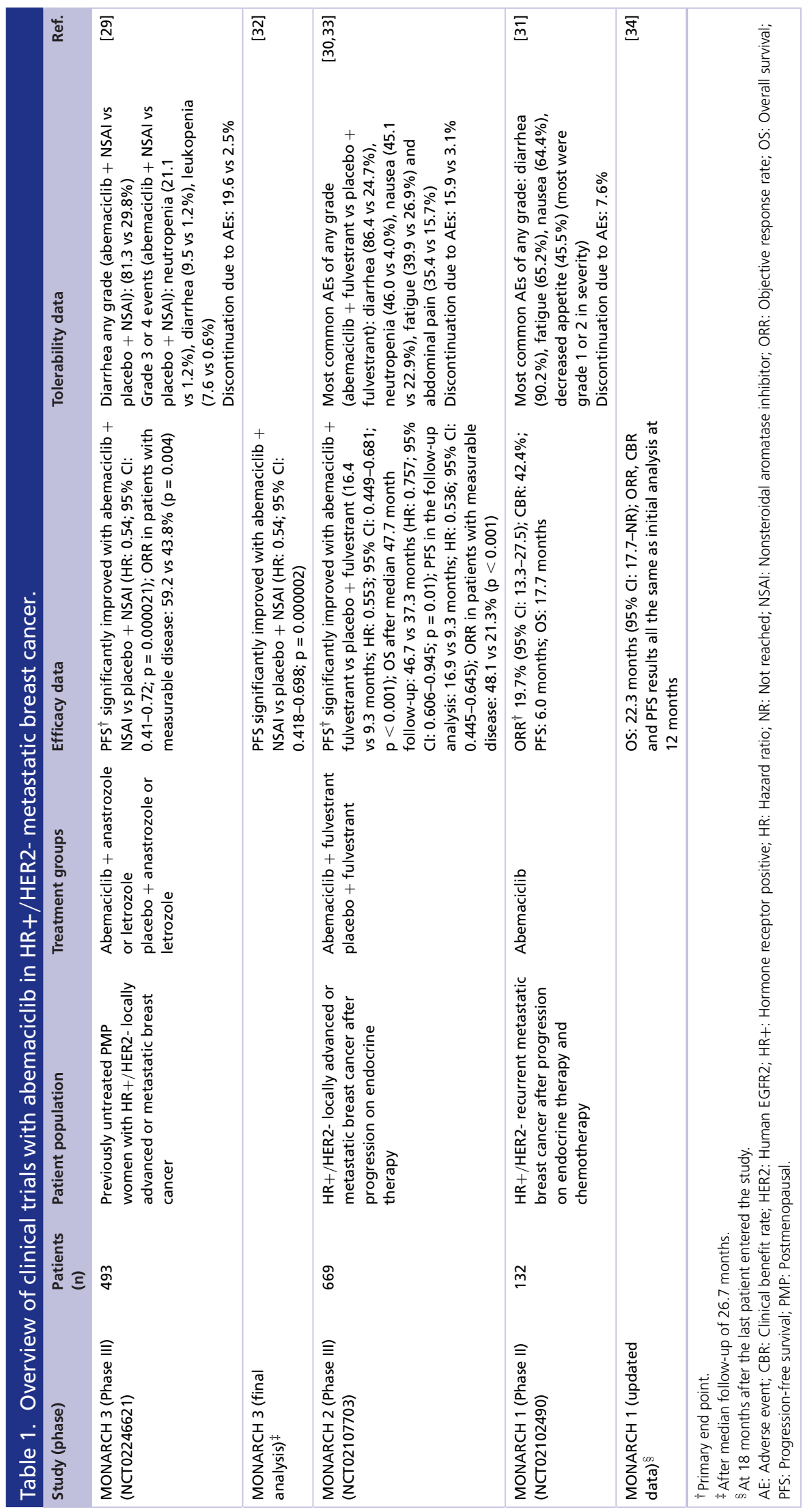


Table 2. Ongoing clinical trials with abemaciclib in breast cancer.

\begin{tabular}{|c|c|c|c|c|}
\hline Study & Patients (n) & Patient population & Treatment groups & $\begin{array}{l}\text { Primary end } \\
\text { point }\end{array}$ \\
\hline \multicolumn{5}{|l|}{ Metastatic breast cancer } \\
\hline $\begin{array}{l}\text { MONARCH plus (Phase III) } \\
\text { (NCT02763566) }\end{array}$ & $463^{\dagger}$ & $\begin{array}{l}\text { Postmenopausal women with HR+/HER2- } \\
\text { locoregionally recurrent or metastatic breast } \\
\text { cancer }\end{array}$ & $\begin{array}{l}\text { Cohort A: abemaciclib + anastrozole or } \\
\text { letrozole vs placebo + anastrozole or letrozole } \\
\text { (first line) } \\
\text { Cohort B: abemaciclib + fulvestrant vs } \\
\text { placebo + fulvestrant (first or second line) }\end{array}$ & PFS \\
\hline $\begin{array}{l}\text { Next MONARCH } 1 \text { (Phase } \\
\text { II) (NCT02747004) }\end{array}$ & $225^{\ddagger}$ & $\begin{array}{l}\text { HR+/HER2- metastatic breast cancer previously } \\
\text { treated with endocrine therapy and } \\
\text { chemotherapy }\end{array}$ & $\begin{array}{l}\text { Abemaciclib + tamoxifen } \\
\text { Abemaciclib } \\
\text { Abemaciclib + prophylactic loperamide }\end{array}$ & PFS \\
\hline $\begin{array}{l}\text { Abema-Pembro (Phase lb) } \\
\text { (NCT02779751) }\end{array}$ & $100 \ddagger$ & $\begin{array}{l}\text { HR+/HER2- metastatic breast cancer previously } \\
\text { treated with chemotherapy } \S\end{array}$ & Abemaciclib + pembrolizumab & $\mathrm{NR}$ \\
\hline $\begin{array}{l}\text { monarcHER (Phase II) } \\
\text { (NCT02675231) }\end{array}$ & $225^{\ddagger}$ & $\begin{array}{l}\text { Postmenopausal women with HR+/HER2+ } \\
\text { locally advanced or metastatic breast cancer } \\
\text { previously treated with HER+-directed therapy }\end{array}$ & $\begin{array}{l}\text { Abemaciclib + fulvestrant + trastuzumab } \\
\text { Abemaciclib + trastuzumab } \\
\text { Trastuzumab + single-agent chemotherapy }\end{array}$ & PFS \\
\hline NCT03130439 (Phase II) & $37 \ddagger$ & $\begin{array}{l}\mathrm{HR}-/ \mathrm{HER} 2-/ \mathrm{Rb}+\text { locally advanced or } \\
\text { recurrent/metastatic breast cancer previously } \\
\text { treated with } 1-3 \text { lines of chemotherapy } \mathbb{I}\end{array}$ & Abemaciclib & ORR \\
\hline \multicolumn{5}{|l|}{ Early-stage breast cancer } \\
\hline $\begin{array}{l}\text { neoMONARCH (Phase II) } \\
\text { (NCT02441946) }\end{array}$ & $224^{\dagger}$ & $\begin{array}{l}\text { Postmenopausal women with HR+/HER2- } \\
\text { early-stage breast cancer }\end{array}$ & $\begin{array}{l}\text { Abemaciclib }+ \text { anastrozole }+ \text { loperamide } \\
\text { Anastrozole } \\
\text { Abemaciclib }+ \text { loperamide }\end{array}$ & Ki67 expression \\
\hline $\begin{array}{l}\text { monarchE (Phase III) } \\
\text { (NCT03155997) }\end{array}$ & $4580^{\ddagger}$ & $\begin{array}{l}\text { High-risk, node-positive women with } \\
\text { HR+/HER2- early-stage breast cancer after } \\
\text { surgery }\end{array}$ & $\begin{array}{l}\text { Abemaciclib + standard adjuvant endocrine } \\
\text { therapy } \\
\text { Standard adjuvant endocrine therapy }\end{array}$ & $\begin{array}{l}\text { Invasive } \\
\text { disease-free } \\
\text { survival }\end{array}$ \\
\hline \multicolumn{5}{|c|}{$\begin{array}{l}\text { †Actual enrollment. } \\
\text { ¥Estimated enrollment. } \\
\text { §Multiple-cohort study with disease indications besides breast cancer. } \\
\text { IDeveloped recurrent/metastatic disease within } 12 \text { months of neoadjuvant/adjuvant chemotherapy. } \\
\text { HER2: Human EGFR2; HR: Hormone receptor; NR: Not reported; ORR: Objective response rate; PFS: Progression-free survival. }\end{array}$} \\
\hline
\end{tabular}

with abemaciclib plus fulvestrant and 37.3 months with placebo plus fulvestrant (HR: 0.757 ; 95\% CI: 0.606$0.945 ; \mathrm{p}=0.01$ ) (Figure 1) [33]. ORR was also significantly greater with combination therapy than with fulvestrant monotherapy in patients with measurable disease ( 48.1 vs $21.3 \%$; $p<0.001)$ [30].

Statistically significant improvements were also seen with abemaciclib plus fulvestrant versus placebo for the exploratory end points of TTC (HR: 0.625; 95\% CI: 0.501-0.779), chemotherapy-free survival (HR: 0.638; 95\% CI: 0.527-0.773) and PFS2 (HR: 0.675; 95\% CI: 0.558-0.816) [33]. In the ITT population, post-discontinuation chemotherapy was received by 30.9 and $43.5 \%$ of women in the abemaciclib plus fulvestrant and placebo plus fulvestrant arms, respectively.

Alongside superior PFS and a manageable safety profile, patient-reported pain and HRQoL outcomes also support treatment with abemaciclib plus fulvestrant in patients with endocrine-resistant HR+/HER2- advanced breast cancer. Only diarrhea significantly favored the control arm (HR: 1.60; 95\% CI: 1.20-2.10) [37]. However, patients receiving abemaciclib had a nonsignificant 4.9-month delay in median time to worsening of pain and a significant delay in time to sustained deterioration on the modified Brief Pain Inventory-short form and in analgesic use (HR: 0.76; 95\% CI: 0.59-0.98) and the QLQ-C30 pain item (HR: 0.62; 95\% CI: 0.48-0.79) [37]. Time to sustained deterioration for functioning and most symptoms significantly favored abemaciclib, including fatigue, nausea and vomiting, and cognitive and social functioning [37].

MONARCH 1 was a Phase II single-arm open-label multinational study that evaluated the efficacy and tolerability of abemaciclib monotherapy in heavily pretreated women with refractory HR+/HER2- locally advanced or metastatic breast cancer [31]. Inclusion criteria were progression on endocrine therapy and at least two lines of chemotherapy, but no more than two lines in the metastatic setting and at least one regimen containing a taxane. Other inclusion criteria were an ECOG performance status of 0 or 1 and measurable disease (RECIST v1.1). The exclusion criteria included a history of brain metastases and previous treatment with another CDK4 and 6 inhibitor. 


\section{(A)}

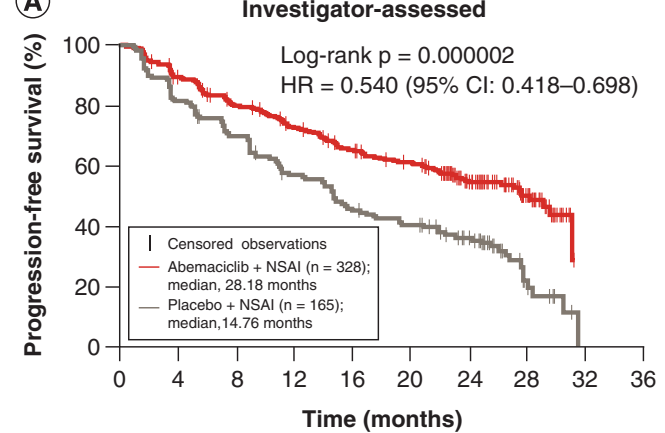

No. at risk: Abemaciclib + NSAI Placebo + NSAI

(C)

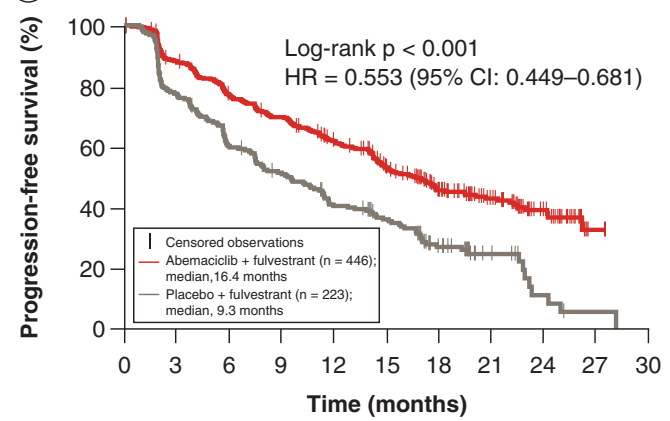

No. at risk:

Abemaciclib + fulvestrant Placebo + fulvestrant $\begin{array}{llllllllll}328 & 272 & 236 & 208 & 181 & 164 & 106 & 40 & 0 & 0\end{array}$ $\begin{array}{llllllllll}165 & 126 & 105 & 84 & 66 & 58 & 42 & 7 & 0 & 0\end{array}$
No. at risk:

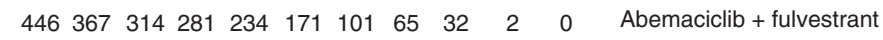
$\begin{array}{lllllllllll}223 & 165 & 123 & 103 & 80 & 61 & 32 & 13 & 4 & 1 & 0\end{array} \quad$ Placebo + fulvestrant

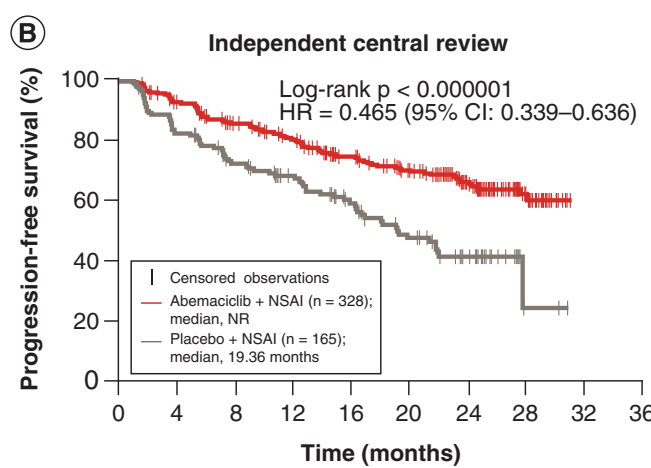

No. at risk: Abemaciclib + NSAI $\begin{array}{llllllllll}328 & 271 & 233 & 206 & 175 & 151 & 98 & 32 & 0 & 0\end{array}$ $\begin{array}{llllllllll}165 & 121 & 96 & 79 & 60 & 44 & 27 & 2 & 0 & 0\end{array}$

(D)

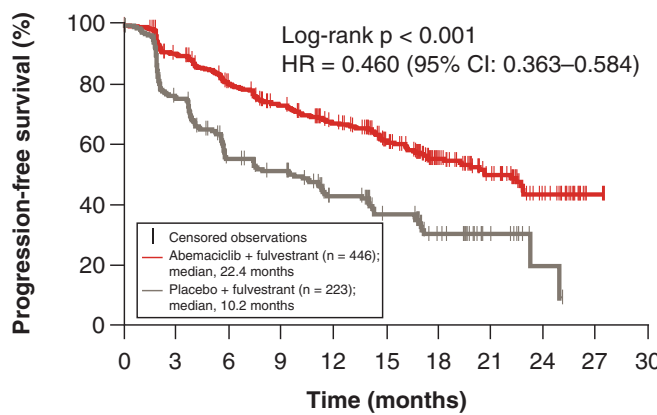

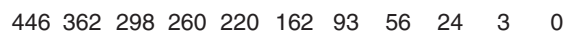

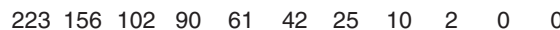

\section{(E) \\ E)}

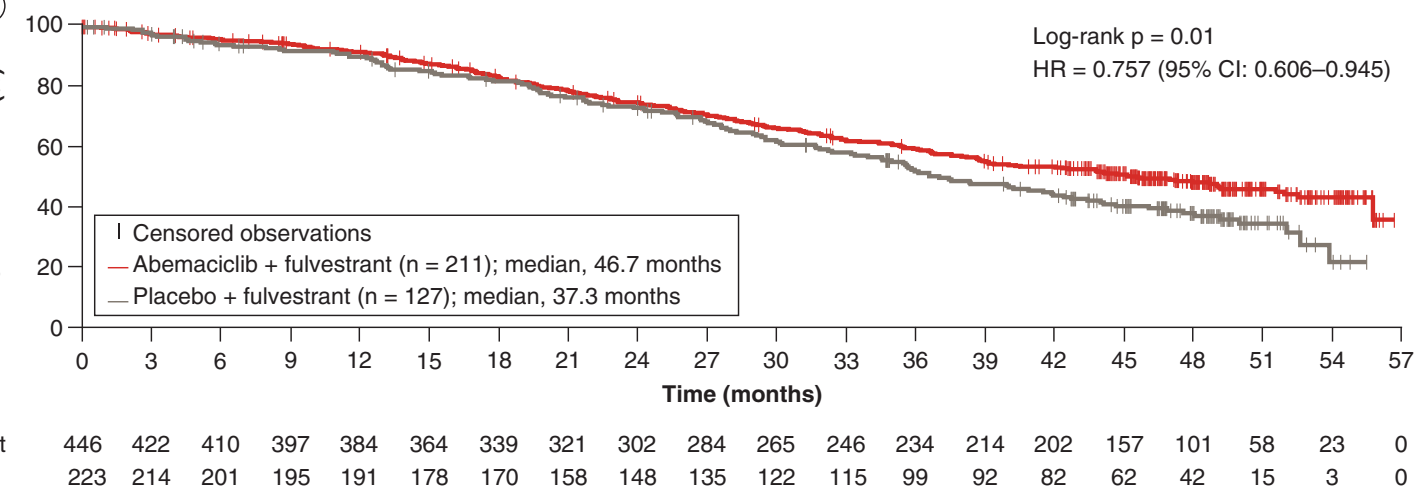

\begin{tabular}{lcccccccccccccccccccc} 
Abemaciclib + fulvestrant & 446 & 422 & 410 & 397 & 384 & 364 & 339 & 321 & 302 & 284 & 265 & 246 & 234 & 214 & 202 & 157 & 101 & 58 & 23 & 0 \\
\hline & 223 & 214 & 201 & 195 & 191 & 178 & 170 & 158 & 148 & 135 & 122 & 115 & 99 & 92 & 82 & 62 & 42 & 15 & 3 & 0
\end{tabular}

$\begin{array}{lllllllllllllllllllll}\text { Placebo + fulvestrant } & 223 & 214 & 201 & 195 & 191 & 178 & 170 & 158 & 148 & 135 & 122 & 115 & 99 & 92 & 82 & 62 & 42 & 15 & 3 & 0\end{array}$

Figure 1. Kaplan-Meier plots of progression-free survival from the MONARCH 3 and 2 trials. PFS from MONARCH 3 according to (A) investigator-assessed and (B) independent central review of the ITT population; PFS from MONARCH 2 according to (C)

investigator-assessed and (D) independent review of the ITT population; and (E) overall survival from MONARCH 2 in the ITT population. HR: Hazard ratio; ITT: Intent-to-treat; NR: Not reached; NSAI: Nonsteroidal aromatase inhibitor; PFS: Progression-free survival. Reproduced with permission from [30,32,33], (c) 2017 American Society of Clinical Oncology.

Abemaciclib was administered in a continuous twice-daily dosing regimen of $200 \mathrm{mg}$ until unacceptable toxicity or disease progression. The primary end point was ORR, with the primary efficacy analysis at 12 months after the last patient entered the trial.

Of the 132 women included in the study, 119 (90.2\%) had visceral metastases and 67 (50.8\%) had three or more metastatic sites [31]. Thus, MONARCH 1 focused on a difficult-to-treat population with indicators of poor prognosis. A primary end point of the trial was ORR statistically designed to exclude an ORR of $\leq 15 \%$ on the lower bound of the $95 \%$ CI at 12 months. No complete responses (CRs) were observed, but 26 women achieved a confirmed partial response (PR), for an ORR of 19.7\% (95\% CI: 13.3-27.5), although the lower bound of the 
95\% CI for the ORR (13.3\%) did not meet the prespecified threshold of 15\% [31]. Among these responders, 12 $(46.2 \%)$ had received at least two prior chemotherapies in the metastatic setting, $12(46.2 \%)$ had at least three metastatic sites and most (92.3\%) had visceral disease. The clinical benefit rate (CBR: CR + PR + stable disease [SD] $\geq 6$ months) was $42.4 \%$ and the median PFS was 6.0 months [31]. Updated data at 18 months after the last patient entered the trial showed no change in ORR (19.7\%; 95\% CI: 13.3-27.5), CBR (42.4\%; 95\% CI: 33.9-51.3), or median PFS (5.95 months; 95\% CI: 4.21-7.50); median OS was 22.3 months (95\% CI: 17.7-not reached) [34].

\section{Safety \& tolerability of abemaciclib}

In general, the AEs associated with abemaciclib in the MONARCH trials were manageable, predictable, and usually reversible with dose reduction.

Across the MONARCH trials, gastrointestinal and hematologic AEs and fatigue were the most frequently reported AEs with abemaciclib (Table 1). Diarrhea typically occurred early in treatment and resolved quickly with conventional regimens of antidiarrheal agents and dose reduction of abemaciclib [30,34]. In the three MONARCH studies, diarrhea was reported in $82.3-90.2 \%$ of abemaciclib recipients, including grade 3 diarrhea in $9.5-19.7 \%$ of patients; grade 4 diarrhea was not reported [35].

A preplanned final analysis of MONARCH 3 data explored the relationship between early diarrhea (within 7 days of initiating study treatment) and PFS. Median PFS in the abemaciclib arm was 28.2 months in those with early diarrhea (HR: 0.49 ; 95\% CI: $0.35-0.67$ ) versus 29.1 months in those without early diarrhea (HR: 0.58 ; 95\% CI: 0.43-0.78) [32]. These results suggest that the development of early diarrhea had little impact on PFS in patients receiving abemaciclib.

In MONARCH 3, dosage reductions due to AEs occurred in $43.4 \%$ of patients receiving abemaciclib compared with $6.2 \%$ of those receiving placebo [29]. Overall, $19.6 \%$ of abemaciclib and $2.5 \%$ of placebo recipients discontinued the study drug because of AEs. The most frequently reported grade 3 or 4 events (abemaciclib plus NSAI vs placebo plus NSAI) were neutropenia ( 21.1 vs $1.2 \%$ ), diarrhea (9.5 vs $1.2 \%$ ) and leukopenia (7.6 vs $0.6 \%)$.

In MONARCH 2, dosage reductions due to AEs occurred in $42.9 \%$ of patients receiving abemaciclib versus $1.3 \%$ of those receiving placebo [30]. Overall, $15.9 \%$ of abemaciclib and $3.1 \%$ of placebo recipients discontinued the study drug because of AEs. The most frequently reported grade 3 or 4 events (abemaciclib plus fulvestrant vs placebo plus fulvestrant) were neutropenia ( 26.5 vs $1.7 \%$ ), diarrhea (13.4 vs $0.4 \%$ ) and leukopenia ( 8.8 vs $0 \%$ ).

Although not frequent, venous thromboembolism events were reported more often with abemaciclib than with placebo in MONARCH 2 (4.8 vs 0.9\%, respectively) and MONARCH 3 (6.1 vs 0.6\%) [35]. However, thromboembolic events are not specific to abemaciclib and have been reported with other CDK4 and 6 inhibitors. A recently reported retrospective analysis by Gervaso et al. in 424 patients treated with CDK 4 and 6 inhibitors (palbociclib in $92 \%$ of cases) showed a 1 -year cumulative incidence of thromboembolic events of $6.3 \%$ [38]. Severe, life-threatening, or fatal interstitial lung disease and/or pneumonitis can occur with abemaciclib and other CDK4 and 6 inhibitors. Across MONARCH 1, 2 and 3, 3.3\% of abemaciclib-treated patients had interstitial lung disease/pneumonitis of any grade, $0.6 \%$ had grade 3 or 4 , and $0.4 \%$ died [39].

Abemaciclib inhibits the renal transporters OCT2, MATE1 and MATE2-K at clinically relevant concentrations, suggesting that abemaciclib may reduce the renal clearance of substrates of these transporters, such as creatinine, without affecting glomerular function [39,40]. In clinical studies with abemaciclib, increases in serum creatinine (mean increase $0.2-0.3 \mathrm{mg} / \mathrm{dl}$ ) occurred within the first 28-day cycle, remained elevated but stable through the treatment period, and were reversible upon treatment discontinuation [39]. However, increases in serum creatinine in patients receiving abemaciclib are not accompanied by an increase in cystatin $\mathrm{C}$, an alternative marker of renal function independent of serum creatinine, and cystatin C-calculated glomerular filtration rate is not reduced [31]. Therefore, the rise in serum creatinine is not temporally associated with reduced renal function and serum creatinine may not provide an accurate reflection of renal function in patients receiving abemaciclib [31,40].

\section{Exploratory subgroup analyses for abemaciclib: efficacy results in prognostic subgroups}

In both MONARCH 3 [29] and MONARCH 2 [30], adding abemaciclib to endocrine therapy provided benefit in the overall study populations, with consistent results across subgroups.

A recent exploratory analysis using data from MONARCH 2 and 3 was conducted to determine independently prognostic subgroups [41]. It also characterized the benefit of the addition of abemaciclib in these subgroups and 
determined those who derived the largest benefit from abemaciclib and those for whom endocrine monotherapy may be an appropriate initial treatment [41].

Significant and independent prognostic characteristics identified in the retrospective analysis of MONARCH 2 and/or 3 included treatment-free interval, liver metastases, bone-only disease, progesterone receptor status, tumor grade, and ECOG performance status [41]. Results showed that all subgroups benefited from the addition of abemaciclib to endocrine therapy in MONARCH 2 and 3, consistent with results in the overall study populations. Among the subgroups analyzed, the greatest effects appeared to occur in patients with more aggressive disease (i.e., treatment-free interval $<36$ months in MONARCH 3; liver metastases, high-grade tumors, or progesterone receptor-negative tumors in MONARCH 2 and 3) [41]. Progesterone receptor-negative disease is usually associated with luminal type $B$ or high proliferative disease $[42,43]$. Figures 2 and 3 show representative results from MONARCH 3 and 2.

Taken together, findings from exploratory analyses of MONARCH 2 and 3 suggest that women with indicators of poor prognosis (including visceral metastases and high proliferative disease) derived greater effects from the addition of abemaciclib to endocrine therapy, although this hypothesis requires further evaluation in prospective studies.

Results of a pooled analysis of five Phase III registration trials of CDK4 and 6 inhibitors conducted by the US FDA are also interesting [44]. They calculated the median PFS and HRs for less common subgroups (progesterone receptor-negative, de novo metastatic and lobular) and found that the benefit was similar to those in the broad population. The investigators reported the natural history of disease and the magnitude of the CDK4 and 6 benefit to be similar for certain subsets previously thought to have different behaviors and considered the results 'hypothesis generating'.

\section{Differential pharmacologic \& clinical characteristics of abemaciclib}

In preclinical studies, prolonged and continuous exposure of estrogen receptor-positive breast cancer cells to abemaciclib resulted in altered cell metabolism and a marked increase in apoptosis and markers of senescence [45]. In addition, observations of the dose escalation part of the Phase I study with abemaciclib indicated that the doselimiting toxicity was grade 3 fatigue [40] in two of three patients at dose levels exceeding the maximum tolerated dose. While neutropenia was observed, it was not the dose-limiting toxicity.

Along with the pharmacokinetic profile of abemaciclib (e.g., elimination half-life of 17.4-38.1 h), these findings established that abemaciclib can be administered safely on a twice-daily (every $12 \mathrm{~h}$ ) continuous dosing schedule [40]. Palbociclib and ribociclib require intermittent administration of 3 weeks on followed by 1 week off $[18,28]$.

One hypothesis for the relatively low risk of severe neutropenia with abemaciclib is its 14-fold greater selectivity for CDK4-cyclin D1 than for CDK6-cyclin D3 observed in cell-free enzymatic assays [45], which contrasts with the ratio of approximately 1.4 for palbociclib [46]; however, ribociclib also has a several-fold greater selectivity for CDK4 over CDK6 [47]. Among the various roles of the different cyclin-dependent kinases, CDK4 and cyclin D1 are important for breast tumorigenesis, whereas CDK6 and cyclin D3 are important for hematopoietic stem cell differentiation [48-54].

Abemaciclib crosses the blood-brain barrier in clinically relevant concentrations, with cerebrospinal fluid concentrations exceeding the inhibitory constant for the CDK4-cyclin D1 complex and approaching unbound plasma abemaciclib concentrations [40]. The recent Phase II JPBO study (ClinicalTrials.gov; NCT02308020) showed that three of the 52 patients in the cohort with brain metastases secondary to HR+/HER2- metastatic breast cancer had a confirmed intracranial response resulting in a $5.8 \%$ objective intracranial response rate, which did not meet the primary objective [55]. However, abemaciclib demonstrated an intracranial CBR of 25\%, and 38\% of patients showed a decrease in the sum of their intracranial target lesion [55]. Further studies are warranted to identify patients with brain metastases secondary to metastatic breast cancer who might benefit most from abemaciclib combinations. A single-arm study of palbociclib in patients with metastatic HER2+ breast cancer with brain metastases is ongoing (NCT02774681).

The activity of abemaciclib monotherapy in heavily pretreated women with HR+/HER2- was demonstrated in MONARCH 1 [31]. In general, CDK4 and 6 inhibitory activity is greater in early lines of therapy and in less-treated patients [56].

With respect to palbociclib monotherapy, a Phase II study showed that, among 37 patients with metastatic breast cancer who had received a median of two prior cytotoxic regimens and were treated with palbociclib, two patients had a PR and five had SD, resulting in a CBR of 19\%; median PFS was 3.7 months [57]. Recent data from the 

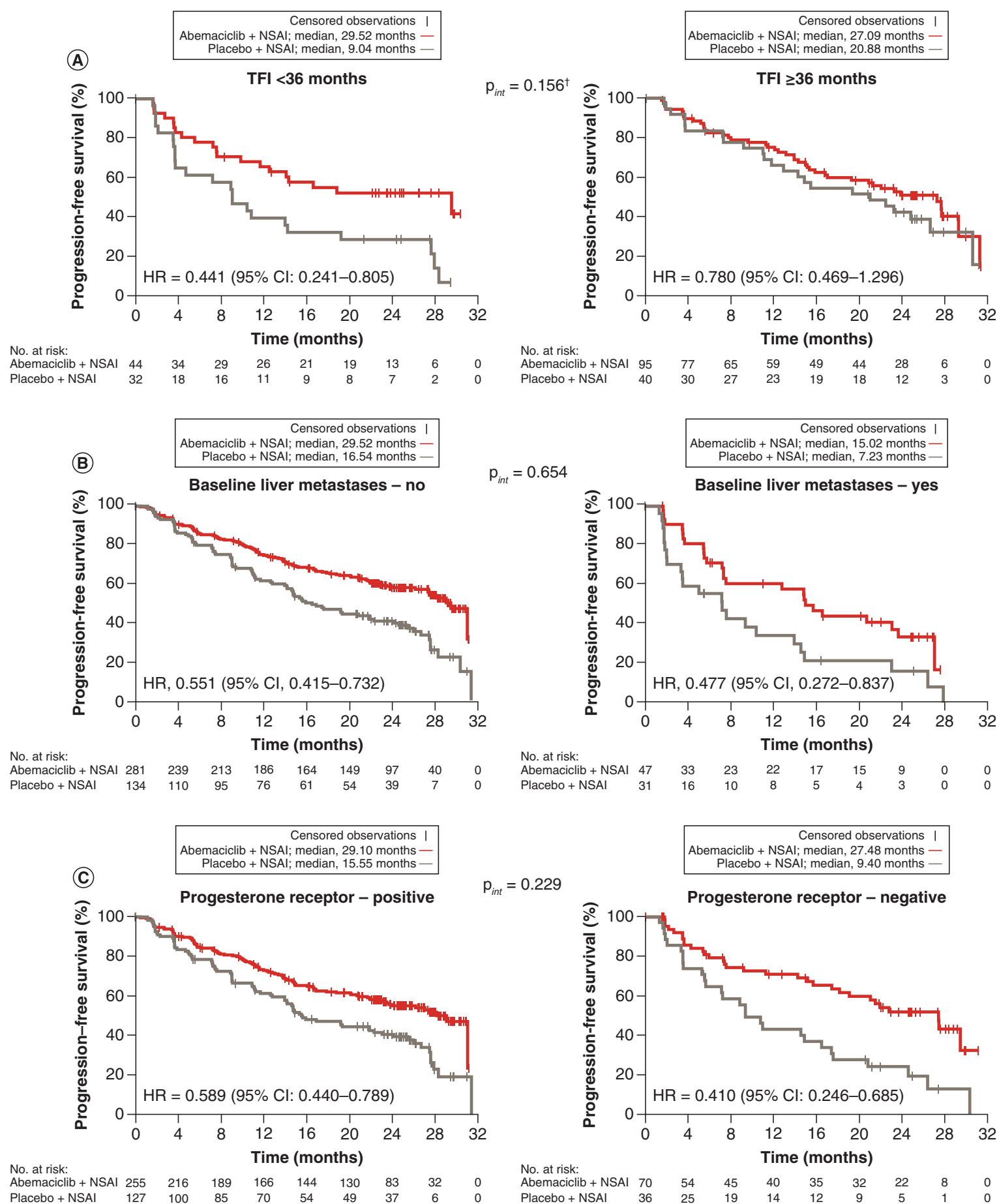

Figure 2. Exploratory subgroup analysis in MONARCH 3 evaluating the efficacy of abemaciclib in patients with characteristics that portend a poor prognosis. (A) Treatment-free interval $<36$ months, (B) liver metastases, and (C) progesterone receptor-negative status.

$\dagger$ Interaction for treatment-free interval has been adjusted for removal of patients with de novo disease.

HR: Hazard ratio; NSAI: Nonsteroidal aromatase inhibitor.

Reproduced from [41]. 

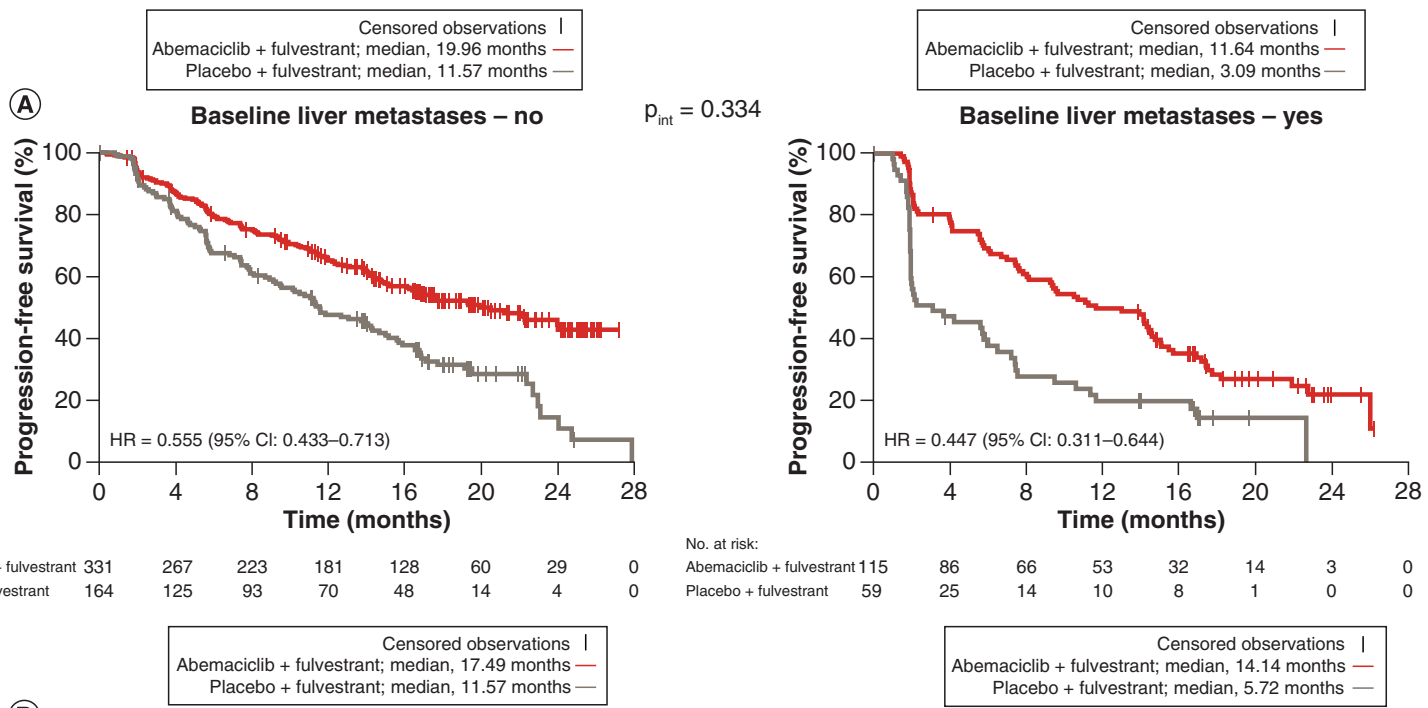

(B)

Censored observations

No. at risk:

$\begin{array}{lllllll}267 & 223 & 181 & 128 & 60 & 29 & 0\end{array}$

Abemaciclib + fulvestrant 331 Placebo + fulvestrant $164 \quad 125$

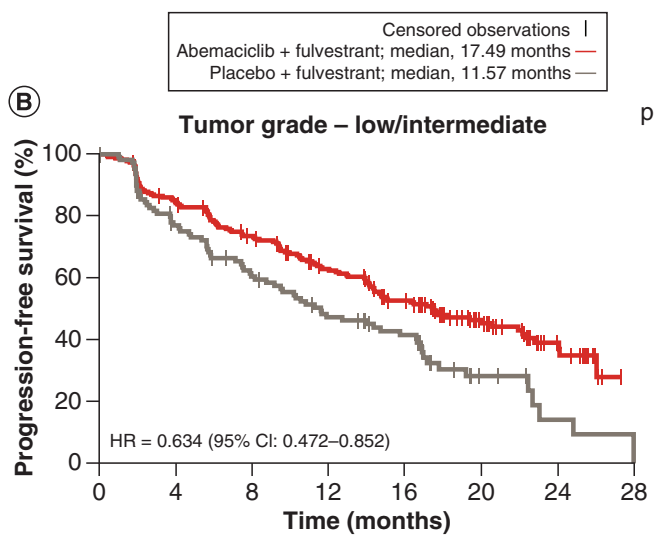

$\mathrm{p}_{\text {int }}=0.263^{\dagger}$

Tumor grade - high

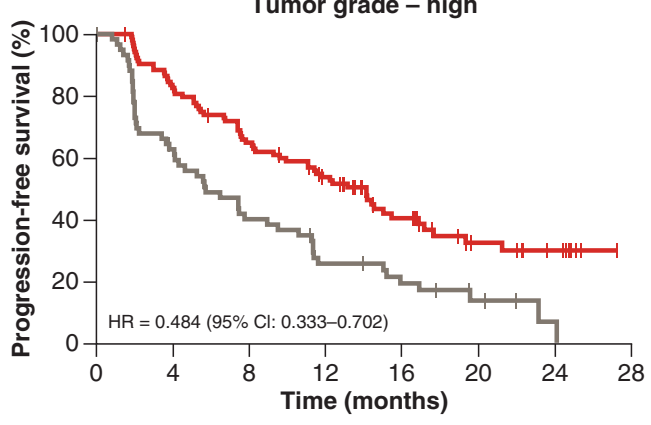

No. at risk:

$\begin{array}{llllllll}\text { Abemaciclib + fulvestrant 109 } & 85 & 65 & 50 & 27 & 13 & 7 & 0\end{array}$

Abemaciclib + fulvestrant

$$
\begin{array}{r}
\text { Censored observations I } \\
\text { Abemaciclib + fulvestrant; median, 16.87 months - } \\
\text { Placebo + fulvestrant; median, 11.24 months - }
\end{array}
$$



$\mathrm{p}_{\mathrm{int}}=0.583$
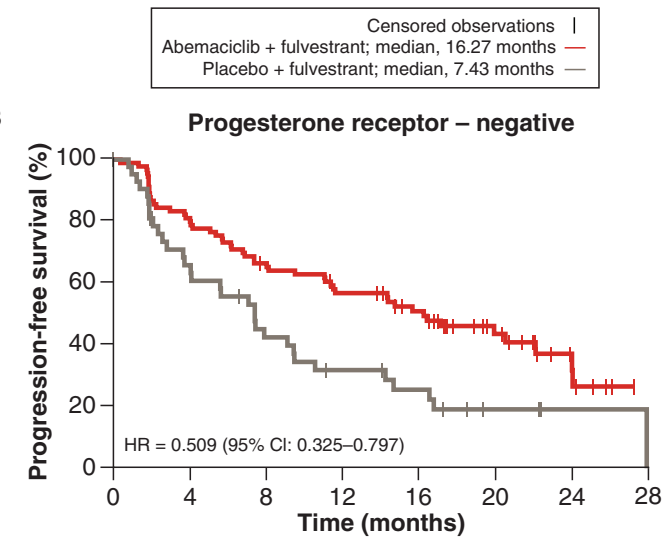

No. at risk:

$\begin{array}{llllllll}\text { Abemaciclib + fulvestrant } 96 & 72 & 56 & 45 & 33 & 17 & 7 & 0\end{array}$

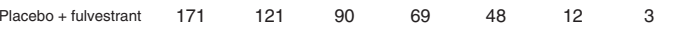

Figure 3. Exploratory subgroup analysis in MONARCH 2 evaluating the efficacy of abemaciclib in patients with characteristics that portend a poor prognosis. (A) Liver metastases, (B) tumor grade, and (C) progesterone receptor-negative status.

$\dagger$ Interaction of tumor grade has been adjusted for removal of patients with unknown tumor grade. HR: Hazard ratio.

Reproduced from [41]. 
Phase II, open-label TREnd trial investigating the role of palbociclib alone or combined with endocrine therapy following progression with the same endocrine therapy provide some information on the efficacy of palbociclib as a single agent in less-pretreated patients [58]. This study enrolled 115 postmenopausal women with estrogen receptor-positive/HER2- advanced breast cancer who progressed after one or two prior endocrine therapies. One previous line of chemotherapy in this setting was allowed. Less than one-third of patients had received two prior endocrine treatments, and approximately one-third of patients in both groups had received prior chemotherapy. The primary end point was $C B R(C R+P R+S D \geq 24$ weeks according to RECIST v1.1 criteria). In the palbociclib monotherapy arm, the CBR (primary end point) was 60\% (95\% CI: 47.8-72.9); at the maximum tolerated dose, the ORR was 7\% and the median PFS was 6.5 months. In addition, findings of a post hoc exploratory analysis in the TREnd trial [58] showed that the duration of clinical benefit significantly favored the combination regimen over palbociclib monotherapy. Comparison of PFS also showed an advantage for the combination arm primarily in the subgroup of patients with a history of endocrine therapy for $>6$ months. These findings suggest that palbociclib may have the potential to reverse endocrine resistance in patients with a previous history of durable endocrine therapy response [58].

\section{Conclusion}

The addition of CDK4 and 6 inhibitors to endocrine therapy, as first-line treatment or following progression after initial endocrine therapy, has become a standard of care in HR+/HER2- metastatic breast cancer. In the key Phase III trials for palbociclib, ribociclib and abemaciclib, the addition of CDK4 and 6 inhibitors to endocrine therapy achieved statistically significant advantages in PFS, which was the primary end point of these studies. In addition, emerging and mature data on OS, an important secondary end point, are also favorable. PALOMA-3 showed a numerical median OS advantage among palbociclib-treated patients and MONALEESA-7 and -3 found statistically significant median OS advantages among ribociclib-treated patients. Recently reported data from MONARCH 2 showed a clinically and statistically significant 9.4-month median OS advantage for abemaciclib-treated patients.

Abemaciclib is administered on a continuous, twice-daily dosing regimen, and its dose-limiting toxicity is fatigue. Abemaciclib also has documented central nervous system penetration, which has led to clinical investigation in patients with brain metastases.

In the Phase III MONARCH 2 and 3 trials, the addition of abemaciclib to endocrine therapy showed a statistically significant and clinically meaningful improvement in PFS compared with placebo plus endocrine therapy in both first- and second-line therapy in women with HR+/HER2- advanced breast cancer (ITT population). Abemaciclib also demonstrated activity as monotherapy in heavily pretreated patients with HR+/HER2- metastatic breast cancer in MONARCH 1. A recent hypothesis-generating exploratory combined analysis of MONARCH 2 and 3 suggested that, among subgroups evaluated, the addition of abemaciclib to endocrine therapy had more pronounced effects in patients with indicators of poor prognosis (i.e., more aggressive disease), namely in women with high proliferative disease, progesterone receptor-negative status, or liver metastases. Given the somewhat contradictory findings of the pooled analysis by the FDA, future research to validate this hypothesis is needed, including identifying biomarkers predictive of treatment benefit of CDK4 and 6 inhibitors, and determining optimal treatment sequencing for different subgroups of patients with HR+/HER2- metastatic breast cancer.

\section{Future perspective}

The addition of CDK4 and 6 inhibitors to endocrine therapy appears to be beneficial across all subgroups of patients with HR+/HER2- metastatic breast cancer. Questions regarding their optimal use include which patients are likely to derive the greatest benefit from treatment, optimal treatment sequencing, the utility of continuing CDK4 and 6 inhibitor therapy after disease progression, as well as the use of these agents in patients with early breast cancer or other subtypes of metastatic breast cancer. Clinical trials with CDK4 and 6 inhibitors (e.g., neoMONARCH and monarcHER with abemaciclib in the neoadjuvant setting and in patients with HR+/HER2+ metastatic breast cancer, respectively) are exploring some of these issues. Findings of a hypothesis-generating exploratory analysis with abemaciclib suggested that patients with HR+/HER2- metastatic breast cancer who have indicators of poor prognosis (i.e., those with more aggressive disease) showed the largest effect. Future and ongoing trials will provide further insight and direction to help guide optimal therapy in clinical practice. 


\section{Background}

- The addition of CDK4 and 6 inhibitors (abemaciclib, palbociclib and ribociclib) to endocrine therapy in patients with hormone receptor-positive $(\mathrm{HR}+)$, HER2- advanced breast cancer aims to reverse or delay endocrine resistance.

Clinical trials with palbociclib, ribociclib \& abemaciclib in HR+/HER2- advanced breast cancer

Palbociclib

- In PALOMA-2, median progression-free survival (PFS) with palbociclib plus letrozole was significantly longer than with placebo plus letrozole ( 24.8 vs 14.5 months; HR: $0.58 ; 95 \% \mathrm{Cl}: 0.46-0.72 ; \mathrm{p}<0.001$ ) as initial therapy in postmenopausal women.

- Median PFS was also significantly improved with palbociclib plus fulvestrant versus placebo plus fulvestrant (9.5 vs 4.6 months; hazard ratio [HR]: 0.46; $95 \% \mathrm{Cl}: 0.36-0.59 ; \mathrm{p}<0.0001$ ) in PALOMA-3, which was conducted in preor postmenopausal women. Numerical (albeit non-significant) increases in median overall survival (OS) were also observed.

\section{Ribociclib}

- MONALEESA-2 evaluated initial treatment in postmenopausal women and showed a statistically significant increase in PFS with ribociclib plus letrozole compared with placebo plus letrozole (25.3 vs 16.0 months; HR: $\left.0.568 ; 95 \% \mathrm{Cl}: 0.457-0.704 ; \mathrm{p}=9.63 \times 10^{-8}\right)$.

- MONALEESA-7 was conducted in premenopausal women in whom previous endocrine therapy was permitted in the neo/adjuvant setting as was chemotherapy in the neo/adjuvant setting or for advanced disease. Median PFS with ribociclib plus endocrine therapy was 23.8 months compared with 13.0 months with placebo plus endocrine therapy (HR: $0.55 ; 95 \% \mathrm{Cl}: 0.44-0.69 ; \mathrm{p}<0.0001)$. An interim analysis also showed a statistically significant advantage for ribociclib over placebo for OS (a secondary end point).

- Ribociclib plus fulvestrant significantly improved PFS (20.5 vs 12.8 months; HR: $0.593 ; 95 \%$ Cl: $0.480-0.732$; $\mathrm{p}<0.001$ ) and OS (a secondary end point) compared with placebo plus fulvestrant in MONALEESA-3, which was conducted in postmenopausal women who were treatment naive or had received up to one line of prior chemotherapy in the advanced setting.

\section{Abemaciclib}

- MONARCH 3 evaluated initial treatment in postmenopausal women and showed a statistically significant increase in PFS with abemaciclib plus nonsteroidal aromatase inhibitor compared with placebo plus nonsteroidal aromatase inhibitor (28.2 vs 14.8 months; HR: $0.540 ; 95 \% \mathrm{Cl}: 0.418-0.698 ; \mathrm{p}=0.000002$ ).

- MONARCH 2 was conducted in pre- or postmenopausal women who had progressed while receiving endocrine therapy. Median PFS was significantly longer with abemaciclib plus fulvestrant than with placebo plus fulvestrant (16.4 vs 9.3 months; HR: $0.553 ; 95 \% \mathrm{Cl}: 0.449-0.681 ; \mathrm{p}<0.001$ ). Updated analysis also showed significantly longer OS (46.7 vs 37.3 months; $p=0.01$ ).

- Abemaciclib also demonstrated efficacy as monotherapy in heavily pretreated women with refractory disease in the Phase II MONARCH 1 trial.

- Findings of a hypothesis-generating exploratory analysis using data from MONARCH 2 and 3 suggested that, among subgroups analyzed, women with indicators of poor prognosis (including visceral metastases and high proliferative disease) derived the greatest benefit from the addition of abemaciclib to endocrine therapy.

\section{Differential pharmacologic \& clinical characteristics of abemaciclib}

- Abemaciclib appears to have a relatively low risk of severe neutropenia. The dose-limiting toxicity of abemaciclib was grade 3 fatigue in a Phase I trial, and the drug can be safely administered on a twice-daily (every $12 \mathrm{~h}$ ) continuous dosing schedule. Palbociclib and ribociclib both require intermittent administration of 3 weeks on followed by 1 week off.

Conclusion

- The addition of CDK4 and 6 inhibitors to endocrine therapy, as first-line treatment or following progression after initial endocrine therapy, is now a standard of care in patients with HR+/HER2- metastatic breast cancer. In Phase III trials with palbociclib, ribociclib and abemaciclib, adding CDK4 and 6 inhibitors to endocrine therapy improved PFS and in some trials also improved OS (an important secondary end point).

- Hypothesis-generating exploratory data for abemaciclib suggest its largest effects may be in subgroups of women with indicators of poor prognosis, although these data require confirmation.

\section{Author contributions}

M Martin performed conceptualization, writing, review and editing. JA Garcia-Saenz performed writing, review and editing. L Manso performed conceptualization, writing, review and editing. A Llombart performed writing, review and editing. A Cassinello performed conceptualization, funding acquisition, supervision, writing, review and editing. M Atienza performed conceptualization, project administration, writing, review and editing. F Ringeisen performed writing, review and editing. E Ciruelos performed conceptualization, writing, review and editing. 
Financial \& competing interests disclosure

This work was funded by Eli Lilly and Company Ltd. M Martin has received personal fees from Amgen, AstraZeneca, Daiichi Sankyo, Eli Lilly, Novartis, Pfizer, Pharmamar, Puma, Roche and Taiho Oncology and research grants from Novartis, Puma and Roche. JA Garcia-Saenz has received personal fees from AstraZeneca, Celgene, Eisai, Eli Lilly and Novartis; travel support from Novartis and Roche; and research funding from AstraZeneca. L Manso has received personal fees from AstraZeneca, Clovis, Eli Lilly, MSD, Novartis, Pfizer and Roche and has received research funding from TESARO. A Llombart has received research funding from Agendia, AstraZeneca, Eisai, Eli Lilly, Genomic Health, Novartis, Pfizer, Pierre Fabre, Roche and TESARO; personal fees from Agendia, Eisai, Eli Lilly, Genomic Health, Novartis, Pfizer and Roche; nonfinancial support from Agendia, AstraZeneca, Novartis, Pfizer and Roche; and is founder and stockholder of MedSIR. A Cassinello and M Atienza are employees of Eli Lilly. F Ringeisen is a former employee of Eli Lilly. E Ciruelos has received personal fees from Eli Lilly, Novartis, Pfizer and Roche. The authors have no other relevant affiliations or financial involvement with any organization or entity with a financial interest in or financial conflict with the subject matter or materials discussed in the manuscript apart from those disclosed.

The authors thank G Plosker and K Goa (Rx Communications, Mold, UK) for medical writing assistance with the preparation of this article, funded by Eli Lilly and Company.

\section{Open access}

This work is licensed under the Attribution-NonCommercial-NoDerivatives 4.0 Unported License. To view a copy of this license, visit http://creativecommons.org/licenses/by-nc-nd/4.0/

\section{References}

Papers of special note have been highlighted as: • of interest; $\bullet \bullet$ of considerable interest

1. Zhang MH, Man HT, Zhao XD, Dong N, Ma SL. Estrogen receptor-positive breast cancer molecular signatures and therapeutic potentials. Biomedical Rep. 2(1), 41-52 (2013).

2. Lal P, Tan LK, Chen B. Correlation of HER-2 status with estrogen and progesterone receptors and histologic features in 3,655 invasive breast carcinomas. Am. J. Clin. Pathol. 123(4), 541-546 (2005).

3. Milani A, Geuna E, Mittica G, Valabrega G. Overcoming endocrine resistance in metastatic breast cancer: current evidence and future directions. World J. Clin. Oncol. 5(5), 990-1001 (2014).

4. Chen P, Lee NV, Hu W et al. Spectrum and degree of CDK drug interactions predicts clinical performance. Mol. Cancer Ther. 15(10), 2273-2281 (2016).

5. de Groot AF, Juijpers CJ, Kroep JR. CDK4/6 inhibition in early and metastatic breast cancer: a review. Cancer Treat. Rev. 60, 130-138 (2017).

6. Hamilton E, Infante JR. Targeting CDK4/6 in patients with cancer. Cancer Treat. Rev. 45, 129-138 (2016).

7. Murphy CG, Dickler MN. The role of CDK4/6 inhibition in breast cancer. Oncologist 20(5), 483-490 (2015).

8. Vidula N, Rugo HS. Cyclin-dependent kinase $4 / 6$ inhibitors for the treatment of breast cancer: a review of preclinical and clinical data. Clin. Breast Cancer 16(1), 8-17 (2016).

9. Komata T, Kanzawa T, Takeuchi H et al. Antitumour effect of cyclin-dependent kinase inhibitors (p16 ${ }^{\mathrm{INK} 4 \mathrm{~A}}, \mathrm{p} 18^{\mathrm{INK} 4 \mathrm{C}}, \mathrm{p} 19^{\mathrm{INK} 4 \mathrm{D}}$, p2 $1^{\mathrm{WAF} 1 / \mathrm{CIP} 1}$ and $\mathrm{p} 27^{\mathrm{KIP} 1}$ ) on malignant glioma cells. Br. J. Cancer 88(8), 1277-1280 (2003).

10. Finn RS, Crown JP, Lang I et al. The cyclin-dependent kinase 4/6 inhibitor palbociclib in combination with letrozole versus letrozole alone as first-line treatment of oestrogen receptor-positive, HER2-negative, advanced breast cancer (PALOMA-1/TRIO-18): a randomised Phase II study. Lancet Oncol. 16(1), 25-35 (2015).

11. Finn RS, Martin M, Rugo HS et al. Palbociclib and letrozole in advanced breast cancer. N. Engl. J. Med. 375(20), 1925-1936 (2016).

- Phase III study (PALOMA-2) that demonstrates improved progression-free survival (PFS) with palbociclib plus letrozole versus placebo plus letrozole as first-line treatment in postmenopausal women with HR+/HER2- advanced breast cancer.

12. Cristofanilli M, Turner NC, Bondarenko I et al. Fulvestrant plus palbociclib versus fulvestrant plus placebo for treatment of hormone-receptor-positive, HER2-negative metastatic breast cancer that progressed on previous endocrine therapy (PALOMA-3): final analysis of the multicentre, double-blind, Phase III randomised controlled trial. Lancet Oncol. 17(4), 425-439 (2016).

- Phase III study (PALOMA-3) that shows improved PFS with palbociclib plus fulvestrant versus placebo plus fulvestrant in preor postmenopausal women with HR+/HER2- metastatic breast cancer who had progressed on previous endocrine therapy.

13. Rugo HS, Finn RS, Diéras V et al. Palbociclib plus letrozole as first-line therapy in estrogen receptor-positive/human epidermal growth factor receptor 2-negative advanced breast cancer with extended follow-up. Breast Cancer Res. Treat. 174(3), 719-729 (2019).

14. Turner NC, Slamon DJ, Ro J et al. Overall survival with palbociclib and fulvestrant in advanced breast cancer. N. Engl. J. Med. 379(20), 1926-1936 (2018). 
15. Cristofanilli M, Slamon DJ, Ro J et al. Overall survival (OS) with palbociclib plus fulvestrant in women with hormone receptor-positive $(\mathrm{HR}+)$, human epidermal growth factor receptor 2-negative (HER2-) advanced breast cancer (ABC) analyses from PALOMA-3 [LBA2_PR]. European Society for Medical Oncology Annual Meeting. Munich, Germany (19-23 October 2018).

16. Harbeck N, Iyer $\mathrm{S}$, Turner $\mathrm{N}$ et al. Quality of life with palbociclib plus fulvestrant in previously treated hormone receptor-positive, HER2-negative metastatic breast cancer: patient-reported outcomes from the PALOMA-3 trial. Ann. Oncol. 27(6), 1047-1054 (2016).

17. Finn RS, Cristofanilli M, Ettl J et al. Treatment effect of palbociclib (PAL) plus endocrine therapy (ET) by prognostic and intrinsic subtype: a joint analysis of PALOMA2 and PALOMA3. J. Clin. Oncol. 36(Suppl. 15), Abstract 1023 (2018).

18. Pfizer, Inc. Palbociclib (Ibrance ${ }^{\circledR}$ ) US prescribing information 2017. (2018). https://www.accessdata.fda.gov/drugsatfda_docs/label/2017/207103s004lbl.pdf

19. Hortobagyi GN, Stemmer SM, Burris HA et al. Ribociclib as first-line therapy for HR-positive, advanced breast cancer. N. Engl. J. Med. 375, 1738-1748 (2016).

- Phase III trial (MONSLEESA-2) that demonstrates improved PFS with ribociclib plus letrozole versus placebo plus letrozole in postmenopausal women with HR+/HER2- metastatic breast cancer who had not previously received systemic therapy for advanced disease.

20. Hortobagyi GN, Stemmer SM, Burris HA et al. Updated results from MONALEESA-2, a Phase III trial of first-line ribociclib plus letrozole versus placebo plus letrozole in hormone receptor-positive, HER2-negative advanced breast cancer. Ann. Oncol. 29(7), 1541-1547 (2018).

21. Verma S, O'Shaughnessy J, Burris HA et al. Health-related quality of life of postmenopausal women with hormone receptor-positive, human epidermal growth factor receptor 2-negative advanced breast cancer treated with ribociclib + letrozole: results from MONALEESA-2. Breast Cancer Res. Treat. 170(3), 535-545 (2018).

22. Tripathy D, Im SA, Colleoni M et al. Ribociclib plus endocrine therapy for premenopausal women with hormone-receptor-positive, advanced breast cancer (MONALEESA-7): a randomised Phase III trial. Lancet Oncol. 19(7), 904-915 (2018).

- Phase III trial (MONALEESA-7) that shows that the addition of ribociclib to endocrine therapy improved PFS in premenopausal women with $\mathrm{HR}+/$ HER2- advanced breast cancer.

23. Im SA, Lu YS, Bardia A et al. Overall survival with ribociclib plus endocrine therapy in breast cancer. N. Engl. J. Med. 381(4), 307-316 (2019).

24. Slamon DJ, Neven P, Chia S et al. Phase III randomized study of ribociclib and fulvestrant in hormone receptor-positive, human epidermal growth factor receptor 2-negative advanced breast cancer: MONALEESA-3. J. Clin. Oncol. 36(24), 2465-2472 (2018).

- Phase III trial (MONALEESA-3) that demonstrates improved PFS with ribociclib plus fulvestrant versus placebo plus fulvestrant in postmenopausal women with HR+/HER2- advanced breast cancer.

25. Fasching PA, Esteva FJ, Pivot X et al. Patient-reported outcomes (PROs) in advanced breast cancer (ABC) treated with ribociclib + fulvestrant: results from MONALEESA-3. Ann. Oncol. Suppl. 8, viii90-viii121 (2018).

26. Slamon DJ, Neven P, Chia $S$ et al. Overall survival (OS) results of the Phase III MONALEESA-3 trial of postmenopausal patients (pts) with hormone receptor-positive $(\mathrm{HR}+)$, human epidermal receptor-positive $(\mathrm{HR}+)$, human epidermal growth factor 2-negative (HER2-) advanced breast cancer (ABC) treated with fulvestrant (FUL) \pm ribociclib (RIB). Ann. Oncol. 30(Suppl. 5), Abstract 7184 (2019).

27. Yardley DA, Chan A, Nusch A et al. Ribociclib + endocrine therapy in patients with hormone receptor-positive, HER2-negative advanced breast cancer presenting with visceral metastases: subgroup analysis of Phase III MONALEESA trials. Cancer Res. 79 (Suppl. 4), Abstract P6-18-07 (2019).

28. Novartis. Ribociclib (Kisqali ${ }^{\circledR}$ ) US prescribing information 2020. (2020). https://www.pharma.us.novartis.com/sites/www.pharma.us.novartis.com/files/kisqali.pdf

29. Goetz MP, Toi M, Campone M et al. MONARCH 3: abemaciclib as initial therapy for advanced breast cancer. J. Clin. Oncol. 35(32), 3638-3646 (2017).

-. Phase III trial (MONARCH 3) that demonstrates improved PFS with abemaciclib plus nonsteroidal aromatase inhibitor versus placebo plus nonsteroidal aromatase inhibitor in postmenopausal women with HR+/HER2- locoregionally recurrent or metastatic breast cancer who had not previously received systemic treatment in this setting.

30. Sledge GW Jr, Toi M et al. MONARCH 2: abemaciclib in combination with fulvestrant in women with HR+/HER2-advanced breast cancer who had progressed while receiving endocrine therapy. J. Clin. Oncol. 35(25), 2875-2884 (2017).

-• Phase III trial (MONARCH 2) that shows improved PFS with abemaciclib plus fulvestrant versus placebo plus fulvestrant in preor postmenopausal women with HR+/HER2- locally advanced or metastatic breast cancer who had progressed while receiving endocrine therapy.

31. Dickler MN, Tolaney SM, Rugo HS et al. MONARCH 1, a Phase II study of abemaciclib, a CDK4 and CDK6 inhibitor, as a single agent in patients with refractory HR+/HER2- metastatic breast cancer. Clin. Cancer Res. 23(17), 5218-5224 (2017).

32. Johnston S, Martin M, Di Leo A et al. MONARCH 3 final PFS: a randomized study of abemaciclib as initial therapy for advanced breast cancer. NPJ Breast Cancer 5, 5 (2019). 
33. Sledge GW, Toi M, Neven P et al. The effect of abemaciclib plus fulvestrant on overall survival in hormone receptor-positive, ERBB2-negative breast cancer that progressed on endocrine therapy - MONARCH 2: a randomized clinical trial. JAMA Oncol. 6(1), $116-124$ (2019).

34. Dickler MN, Tolaney SM, Rugo HS et al. MONARCH 1, a Phase 2 study of abemaciclib, a CDK4 and CDK6 inhibitor, as a single agent, in patients with refractory HR+/HER2- metastatic breast cancer. American Association for Cancer Research Annual Meeting. Washington DC, USA, 1-5 April 2017 (Poster).

35. European Medicines Agency. Abemaciclib (verzenios) assessment report 26 July 2018, EMA/551438/2018. (2019). https://www.ema.europa.eu/en/medicines/human/EPAR/verzenios

36. Tolaney SM, Di Leo A, Llombart-Cussac A et al. Impact of abemaciclib on the time to subsequent chemotherapy and the time to second disease progression across the MONARCH 2 and 3 studies. American Society of Clinical Oncology Annual Meeting. IL, USA, 1-5 June 2018 (Poster 1048).

37. Kaufman PA, Toi M, Neven P et al. Health-related quality of life in MONARCH 2: abemaciclib plus fulvestrant in hormone receptor-positive, HER2-negative advanced breast cancer after endocrine therapy. Oncologist 25(2), e243-e251 (2020).

38. Gervaso L, Montero AJ, Jia X, Khorana AA. Venous thromboembolism in breast cancer patients receiving cyclin-dependent kinase inhibitors. J. Thromb. Haemost. 18(1), 162-168 (2020).

39. Eli Lilly and Company, Ltd. Abemaciclib (verzenio $\left.{ }^{\circledR}\right)$ US prescribing information, September 2019. (2019). http://pi.lilly.com/us/verzenio-uspi.pdf

40. Patnaik A, Rosen LS, Tolaney SM et al. Efficacy and safety of abemaciclib, an inhibitor of CDK4 and CDK6, for patients with breast cancer, non-small cell lung cancer and other solid tumors. Cancer Discov. 6(7), 740-753 (2016).

41. Di Leo A, O’Shaughnessy J, Sledge GW Jr et al. Prognostic characteristics in hormone receptor-positive advanced breast cancer and characterization of abemaciclib efficacy. NPJ Breast Cancer 4, 41 (2018).

42. Inic Z, Zegarac M, Inic M et al. Difference between luminal A and luminal B subtypes according to Ki-67, tumor size and progesterone receptor negativity providing prognostic information. Clin. Med. Insights Oncol. 8, 107-111 (2014).

43. Prat A, Cheang MCU, Martin M et al. Prognostic significance of progesterone receptor-positive tumor cells within immunohistochemically defined luminal A breast cancer. J. Clin. Oncol. 31(2), 203-209 (2013).

44. Gao JJ, Cheng J, Bloomquist E et al. Benefit of CDK 4/6 inhibition in less common breast cancer subsets: a U.S. Food and Drug Administration pooled analysis. J. Clin. Oncol. 36 (Suppl. 15), Abstract 1024 (2018).

45. Torres-Guzmán R, Calsina B, Hermoso A et al. Preclinical characterization of abemaciclib in hormone receptor positive breast cancer. Oncotarget 8(41), 69493-69507 (2017).

46. De Dios A, Lallena M, Buchanan SG, Beckmann RP. Mechanisms of action of the CDK4 and CDK6 inhibitor abemaciclib: single-agent and combination activities in preclinical models for human cancer. Presented at: American Association for Cancer Research Annual Meeting. LA, USA, 16-20 April 2016.

47. Chang JC, O'Regan R. Use of novel combination therapies in the treatment of advanced HR+/HER2- breast cancer. J. Natl Compr. Canc. Netw. 16(Suppl. 1), S1-S14 (2018).

48. Caldon CE, Daly RJ, Sutherland RL, Musgrove EA. Cell cycle control in breast cancer cells. J. Cell. Biochem. 97(2), 261-274 (2006).

49. Finn RS, Dering J, Conklin D et al. PD 0332991, a selective cyclin D kinase 4/6 inhibitor, preferentially inhibits proliferation of luminal estrogen receptor-positive human breast cancer cell lines in vitro. Breast Cancer Res. 11(5), R77 (2009).

50. Laurenti E, Frelin C, Xie S et al. CDK6 levels regulate quiescence exit in human hematopoietic stem cells. Cell Stem Cell 16(3), 302-313 (2015).

51. Malumbres M, Barbacid M. Cell cycle, CDKs and cancer: a changing paradigm. Nat. Rev. Cancer 9(3), 153-166 (2009).

52. Scheicher R, Hoelbl-Kovacic A, Bellutti F et al. CDK6 as a key regulator of hematopoietic and leukemic stem cell activation. Blood 125(1), 90-101 (2015).

53. Yu Q, Geng Y, Sicinski P. Specific protection against breast cancers by cyclin D1 ablation. Nature 411(6841), 1017-1021 (2001).

54. Yu Q, Sicinska E, Geng Y et al. Requirement for CDK4 kinase function in breast cancer. Cancer Cell 9(1), $23-32$ (2006).

55. Anders CK, Le Rhun E, Bachelot TD et al. A Phase II study of abemaciclib in patients (pts) with brain metastases (BM) secondary to HR+, HER2- metastatic breast cancer (MBC). J. Clin. Oncol. 37 (Suppl. 15), Abstract 1017 (2019).

56. Deng Y, Ma G, Li W, Wang T, Zhao Y, Wu Q. CDK4/6 Inhibitors in combination with hormone therapy for HR+/HER2-advanced breast cancer: a systematic review and meta-analysis of randomized controlled trials. Clin. Breast Cancer 18(5), e943-e953 (2018).

57. DeMichele A, Clark AS, Tan KS et al. CDK 4/6 inhibitor palbociclib (PD0332991) in Rb+ advanced breast cancer: Phase II activity, safety and predictive biomarker assessment. Clin. Cancer Res. 21(5), 995-1001 (2015).

58. Malorni L, Curigliano G, Minisini AM et al. Palbociclib as single agent or in combination with the endocrine therapy received before disease progression for estrogen receptor-positive, HER2-negative metastatic breast cancer: TREnd trial. Ann. Oncol. 29(8), 1748-1754 (2018). 